\title{
Customized exogenous ferredoxin functions as an efficient electron carrier
}

\author{
Zhan Song ${ }^{1}$, Cancan $\mathrm{Wei}^{1}$, Chao $\mathrm{Li}^{1}$, Xin Gao ${ }^{1}$, Shuhong Mao ${ }^{1}$, Fuping $\mathrm{Lu}^{1}$, and Hui-Min \\ Qin $^{1}$
}

${ }^{1}$ Tianjin University of Science and Technology - Tianjin Economic and Technological Development Area Campus

September 24, 2021

\begin{abstract}
Ferredoxin $(\mathrm{Fdx})$ is regarded as the main electron carrier in biological electron transfer and acts as an electron donor in metabolic pathways of many organisms. Here, we screened a self-sufficient P450-derived reductase PRF with promising NADPH reduction activity and 9OHAD production yield and proved the importance of [2Fe-2S] clusters of Fdx-containing oxidoreductase in transferring electrons in steroidal conversion. The truncated Fdx domain in all oxidoreductases, together with mutagenesis data, further elucidated the indispensable role of $[2 \mathrm{Fe}-2 \mathrm{~S}]$ clusters in the electron transfer process. By adding the independent plant-type Fdx to the reaction system, the AD conversion rate have been significantly improved. A novel efficient electron transfer pathway of $\mathrm{PRF}+\mathrm{Fdx}+\mathrm{KshA}$ in the reaction system rather than KshAB complex system was proposed based on analysis of protein-protein interactions and redox potential measurement. Adding free Fdx created a new conduit for electrons to travel from reductase to oxygenase. This electron transfer pathway provides new insight for the development of efficient exogenous Fdx as an electron carrier.
\end{abstract}

\section{Customized exogenous ferredoxin functions as an efficient electron carrier}

Zhan Song ${ }^{1}$, Cancan $\mathrm{Wei}^{1}$, Chao $\mathrm{Li}^{1}$, Xin $\mathrm{Gao}^{1}$, Shuhong Mao ${ }^{1, *}$, Fuping Lu ${ }^{1, *}$, and Hui-Min Qin ${ }^{1, *}$

${ }^{1}$ Key Laboratory of Industrial Fermentation Microbiology of the Ministry of Education; Tianjin Key Laboratory of Industrial Microbiology; College of Biotechnology, Tianjin University of Science and Technology; National Engineering Laboratory for Industrial Enzymes; Tianjin 300457, P. R. China

*Corresponding authors: College of Biotechnology, Tianjin University of Science and Technology,

S. Mao: shuhongmao@tust.edu.cn;

F. Lu: lfp@tust.edu.cn;

H.-M. Qin: huiminqin@tust.edu.cn.

Tel: +86-22-60602949. Fax: +86-22-60602298

\section{ABSTRACT}

Ferredoxin $(\mathrm{Fdx})$ is regarded as the main electron carrier in biological electron transfer and acts as an electron donor in metabolic pathways of many organisms. Here, we screened a self-sufficient P450-derived reductase PRF with promising NADPH reduction activity and 9OHAD production yield and proved the importance of [2Fe-2S] clusters of Fdx-containing oxidoreductase in transferring electrons in steroidal conversion. The truncated $\mathrm{Fdx}$ domain in all oxidoreductases, together with mutagenesis data, further elucidated the indispensable role of [2Fe-2S] clusters in the electron transfer process. By adding the independent plant-type Fdx 
to the reaction system, the $\mathrm{AD}$ conversion rate have been significantly improved. A novel efficient electron transfer pathway of $\mathrm{PRF}+\mathrm{Fdx}+\mathrm{KshA}$ in the reaction system rather than KshAB complex system was proposed based on analysis of protein-protein interactions and redox potential measurement. Adding free Fdx created a new conduit for electrons to travel from reductase to oxygenase. This electron transfer pathway provides new insight for the development of efficient exogenous Fdx as an electron carrier.

KEYWORDS: ferredoxin; electron transfer; electron bifurcation; [2Fe-2S] clusters

\section{INTRODUCTION}

Ferredoxin (Fdx) was firstly discovered and isolated from Clostridium pastoris (a non-photosynthetic anaerobic bacteria) by Mortenson et al. in 1962 (Mortenson et al., 1962). It is a small metalloprotein with [2Fe-2S] clusters as redox centers (Arnon, 1988). They play a key role as electron carriers in various metabolic processes (Huang et al., 2016). In non-photosynthetic organisms, electron transfer is mediated by NAD $(\mathrm{P}) \mathrm{H}$ (Arnon, 1965; Goss \& Hanke, 2014; Martı'nez-Espinosa et al., 2003). The Fe-S cluster in Fdx physiologically transfers only one electron from $\mathrm{NAD}(\mathrm{P}) \mathrm{H}$, and the reduced Fdx can drive the reaction more strongly because its redox potential is lower than that of $\mathrm{NAD}(\mathrm{P}) \mathrm{H}$ (Kameya et al., 2020). Fdx has been classified as [2Fe-2S], [4Fe-4S], [3Fe-4S] and [7Fe-8S] (Aono et al., 1992; Imai et al., 2001; Lovgreen et al., 2011; Ricagno et al., 2007), in which [2Fe-2S] clusters are divided into two types: plant-type and Rieske-type. In plant-type Fdxs, the $[2 \mathrm{Fe}-2 \mathrm{~S}]$ clusters are organized as $[2 \mathrm{Fe}-2 \mathrm{~S}]$ tetrahedrons, containing two inorganic $\mathrm{Fe}$ atoms and sulfurs from four conserved cysteine residues. In Rieske-type Fdxs, they are composed of inorganic iron atoms, two cysteine and histidine residues. Importantly, plant-type Fdx plays a central role in the electron transfer between the photosynthesis chain and various metabolic pathways (Bohme, 1977; Kimata-Ariga et al., 2006; Lee et al., 2011).

Previous research indicated that $\mathrm{Fdx}$ and $\mathrm{Fdx}_{-\mathrm{NADP}}{ }^{+}$-reductase $(\mathrm{Fdx} / \mathrm{FNR})$ could form complex during electron transfer process (Bohme, 1977; Hurley et al., 1997; Kimata-Ariga et al., 2006; Lee et al., 2011; Quaranta et al., 2016). The functional and structural properties of Fdx/FNR interaction have been investigated suitable redox partners are needed to carry out effectively electron transfer serving as intermediates in steroidal conversion catalyzed by P450 enzymes (Kimata-Ariga et al., 2007; Quaranta et al., 2016; Ueno et al., 2013). Studies have established the principle of customizing non-physiological Fdx to support similar native P450 activity (Bell et al., 2012). The addition of the [2Fe-2S] binding domain in the N-terminus of $\mathrm{KshB}$ (Fdx reductase of 3-ketosteroid-9-hydroxylase) or TDO (toluene 2,3-dioxygenase reductase) was also reported for efficiently strengthening electron transfer by [2Fe-2S] Fdx (Zhu et al., 2020). The electron transfer pathway was proposed from NADH via FAD to the terminal active-site mononuclear iron of the dioxygenase in the reductase and the Fdx protein by elucidating the molecular mechanism of the redoxdependent interaction between NADH-dependent reductase and Rieske-type [2Fe-2S] Fdx (Zhu et al., 2020). The structures of TDO including TDO-R (3EF6) and TDO-F (3DQY), and BphA4-BphA3 complex (FADNADH containing Fdx reductase BphA4 and Rieske-type [2Fe-2S] Fdx BphA3 in the electron transfer system of biphenyl dioxygenase BphA) were reported previously (Senda et al., 2007) that the reductase BphA4 receives two electrons from NADH. The reduced BphA4-BphA3 complex then transfers an electron to the [2Fe-2S] clusters of two Fdx molecules through redox-dependent transient interactions. The reduced Fdx transfers the electron to the active center of the oxygenase to support the activation of dioxygen for biphenyl dihydroxylation. Self-sufficient P450 monooxygenases are highly active and convenient systems without partner reductase (Chen et al., 2020). The crystal structure of the self-sufficient P450 enzyme CYP116B46 has indicated that it contains the cofactor FMN to capture electrons from NADPH and RP Fdx, which has most efficient electron transfer. The direction of electron transfer NADPH-FMN-[2Fe-2S] -heme correlates with the domain arrangement, which undoubtedly provides a great help for the screening of redox partners (Zhang et al., 2020). Otherwise, Based on the flavin-based electron bifurcation theory (FBEB), the electron bifurcation phenomenon is formed by the oxidation of NADPH in the oxidoreductase complex (Demmer et al., 2015; Duan et al., 2020; Lubner et al., 2017; Schut et al., 2019; Tokmina-Lukaszewska et al., 2018; Yuly et al., 2019). The FBEB bifurcation reaction forms a highly unstable flavonoid anion semiquinone (ASQ) to reduce Fdx (Kremp et al., 2020; Sucharitakul et al., 2020). The key step is to transfer electrons from a 
molecule with a medium reduction potential to one of the lower potentials (thermodynamically unfavorable) by coupling the electrons with the transfer to the high potential acceptor (thermodynamically favorable) (Miller et al., 2019; Muller et al., 2018; Schut et al., 2019). Fdx is the electron carrier requiring for all reactions catalyzed by electron bifurcation enzymes (Huang et al., 2016; Liang et al., 2019).

Here, we screened and characterized the reductases involving plant-type and Reiske-type Fdxs. A promising reductase of partial self-sufficient P450 CYP116B46 (V465-L779) from Tepidiphilus thermophiles, named PRF, which contains the flavin mononucleotide (FMN)-binding reductase and [2Fe-2S] clusters was characterized. We added the independent Fdxs to the reaction system instead of KshAB to improve the conversion rate of $\mathrm{AD}$ to $9 \mathrm{OHAD}$. A novel electron transfer pathway was proposed using exogenous Fdx as an efficient electron carrier based on analysis of protein-protein interactions and redox potential measurement.

\section{MATERIALS AND METHODS}

\subsection{Plasmids construction, expression and purification of enzymes}

The coding sequences for the reductase domain and Fdx (V465-L779) ofTepidiphilus thermophilusCYP116B46 (PRF) (WP_055423153.1), Thermosynechococcus elongatus BP-1 Fdx (TeFdx) (WP _011056851.1),Drosophila melanogaster Fdx1 (DmFdx1) and Fdx2 (DmFdx2) (BI626767.1 and BI168285.1), Pseudomonas resinovorans $\mathrm{Fdx}(\mathrm{PrFdx})$ (WP_011077882.1), Pseudomonas sp. Fdx (PsFdx1) (WP 015014864.1), Sphingobium yanoikuyae Fdx (SyFdx) (WP_025548169.1), were synthesized by GENEWZ (Suzhou, China) and cloned into the vector pET28a $(+)$ between the NdeI and EcoRI restriction sites. KshA (Rieske-type oxygenase of 3-ketosteroid-9-hydroxylase) from Rhodococcus erythropolis, KshB (a Fdx reductase of 3-ketosteroid-9-hydroxylase) from Mycobacteriumtuberculosis H37Rv, TDO_R (toluene 2,3-dioxygenase) fromPseudomonas putida were kept in the laboratory. The recombinant plasmid was introduced into Escherichia coli strains JM109 and BL21(DE3) as the host organism for gene cloning and protein expression, were purchased from Novagen (Madison, WI, USA). The strains, plasmids, and primers used in this study are listed in Tables S1. Bacteria were grown with shaking in lysogeny broth medium containing $40 \mu \mathrm{g} / \mathrm{mL}$ kanamycin at $37^{\circ} \mathrm{C}$. When the $\mathrm{OD}_{600}$ reached $0.6-0.8,0.1 \mathrm{mM}$ isopropyl- $\beta$-D-thiogalactopyranoside (IPTG) was added to induce the overexpression of enzymes at $16{ }^{\circ} \mathrm{C}$ for $16-18 \mathrm{~h}$. Then the bacteria were collected by centrifugation at $5,000 \times g$ for $15 \mathrm{~min}$ at $4{ }^{\circ} \mathrm{C}$, and resuspended in the lysis buffer $(20 \mathrm{mM}$ Tris-HCl, 40 $\mathrm{mM}$ imidazole, $0.5 \mathrm{M} \mathrm{NaCl}, 1 \mathrm{mM}$ DTT, $\mathrm{pH}$ 7.4), lysozyme can be added to improve cell disruption. After ultrasonic disruption, the supernatant was collected by centrifugation at $40,000 \times g$ for $30 \mathrm{~min}$ at $4{ }^{\circ} \mathrm{C}$ and the $\mathrm{His}_{6}$-tagged enzymes were trapped on Ni-NTA Superflow resin (Qiagen, Hilden, Germany). The enzymes were eluted by elution buffer ( $20 \mathrm{mM}$ Tris-HCl, $400 \mathrm{mM}$ imidazole, $0.5 \mathrm{M} \mathrm{NaCl}, 1 \mathrm{mM}$ DTT, pH 7.4), and dialyzed against $20 \mathrm{mM}$ HEPES, pH 7.4 finally. The fractions were further purified using anion exchange chromatography on a Resource Q column (column volume: $6 \mathrm{~mL}$, flow rate: $4 \mathrm{~mL} / \mathrm{min}$, GE Healthcare, Stockholm, Sweden).

\subsection{Activity assays}

The NADH reduction activity was monitored by following UV absorption at $340 \mathrm{~nm}$ using a multimode plate reader (SpectraMax i3x, Molecular Devices, Silicon Valley, CA, USA). The specific operation method can refer to our existing research. The Michaelis-Menten constants $\left(K_{\mathrm{m}}\right)$, turnover number $\left(k_{\text {cat }}\right)$, catalytic efficiencies $\left(k_{\text {cat }} / K_{\mathrm{m}}\right)$ for each substrate were calculated by the Michaelis-Menten function in the GraphPad Prism (GraphPad Software, San Diego, CA, USA).

\subsection{Multienzyme cascade catalysis in vitro}

The cell-free multienzyme catalysis was performed and mixing with $50 \mathrm{mM}$ PBS (pH 7.4), $200 \mu \mathrm{M} \mathrm{AD}$ dissolved in $2 \%$ methanol, $300 \mu \mathrm{M} \mathrm{NAD}(\mathrm{P}) \mathrm{H}$, and enzymes $(5 \mu \mathrm{M}$ reductases, $5 \mu \mathrm{M}$ Fdxs, and $2.5 \mu \mathrm{M}$ KshA). The HPLC was used to determine the yield of 9OHAD (Zhu et al., 2020). The strains used are listed in Tables S1, plasmids and primers used in this study are listed in Tables S2.

\subsection{Site-directed mutagenesis}


All variants described in this work were generated using the KOD-Plus Site-Directed Mutagenesis Kit (Toyobo, Japan) and the pET-28a (+) or pCold I vector harboring the wild-type sequence. The oligonucleotides designed to introduce the mutations are depicted in Table S2. All mutations were confirmed by DNA sequencing.

\subsection{Spectroscopic absorption}

All UV-vis spectroscopic absorption experiments were performed with Agilent Cary 2500 UV-visible Spectrophotometer (Agilent Technologies, Beijing, China) at $25{ }^{\circ} \mathrm{C}$. The enzymes were dialyzed against the same buffer (20 mM HEPES, pH 7.4). The spectral absorbance of $20 \mathrm{mM}$ HEPES buffer was scanned in the range of $300-800 \mathrm{~nm}$ as a blank control, and then the UV-vis spectral absorbances of the oxidized state enzymes were measured. $100 \mathrm{mM}$ sodium dithionite was added into the samples until there was no further decrease in absorbance in the 300-800 $\mathrm{nm}$ range and the spectrum of the reduced state enzymes was measured.

\subsection{Homology modeling}

A three-dimensional (3D) homology model were constructed using automodel command in Modeller 9.9.2, with the crystal structure of Rhodococcus rhodochrous 3-ketosteroid-9-alpha-hydroxylase 5 (PDB ID: 4QDC, $75.38 \%$ sequence identity with KshA), Haloarcula marismortui $\mathrm{Fdx}$ (PDB ID: 1DOI, $32.14 \%$ sequence identity with KshB-Fdx), human Fdx 2 (PDB ID: 2Y5C, 63.21\% sequence identity with DmFdx1), and urate oxidase (PDB ID: 3PLM, 61.74\% sequence identity with DmFdx2) as the template. The DOPE assessment scores were used to choose the best model. The model structures were visualized by PyMOL software (http://www.pymol.org).

\subsection{Protein-protein docking}

All protein-protein docking used the website http://zdock.umassmed.edu/. The oxygenase-reductase complex was first obtained. The Fdx was then docked to the oxygenase-reductase complex, and obtained the best results by manually analysis the best top 10 results obtained on website http://zdock.umassmed.edu/. The subsequent processing and analysis are carried out using the PyMOL software (http://www.pymol.org).

\subsection{ITC measurement}

The affinity of ferredoxin DmFdx2 with oxygenase KshA and reductase PRF was determined by ITC 200 instrument (MicroCal, USA) at $25{ }^{\circ} \mathrm{C}$. Each sample solution was dialyzed against the same buffer (20 mM HEPES, pH 7.4) and degassed under vacuum before calorimetry. The reaction cell and injection syringe were filled with $0.02 \mathrm{mM} \mathrm{KshA} / \mathrm{PRF}$ and $0.2 \mathrm{mM} \mathrm{DmFdx} 2$, respectively. The reaction temperature, stirring speed, and reference power were set at $25{ }^{\circ} \mathrm{C}, 800 \mathrm{rpm}$, and $5.00 \mu \mathrm{cal} / \mathrm{s}$, respectively. An aliquot comprising $2 \mu \mathrm{L}$ of the $\mathrm{DmFdx} 2$ solution was added per injection, and each injection cycle lasted for $120 \mathrm{~s}$. The interval between two injections was $60 \mathrm{~s}$ to allow for the signal to return to the baseline. The data were analyzed using Origin 7.0 software, and the data were fitted to the Wiseman equation using a one-site binding model. The stoichiometry $(n)$, binding constant $\left(K_{D}\right)$, binding enthalpy $\left({ }^{H}\right)$, andentropychange $\left({ }^{S}\right)$ couldbedetermined.ThebindingGibbsenergychange ${ }^{G}$, iscalculatedfromtheequations ${ }^{G}=$ $-R \operatorname{Tln} K_{D}$.

\subsection{Redox potential measurement}

The electrochemical titration experiments were performed using Mettler T50 Automatic Potentiometric Titrator with an $\mathrm{Ag} / \mathrm{AgCl}$ electrode at $25{ }^{\circ} \mathrm{C}$. The $25 \mu \mathrm{M}$ protein samples were dispersed in Tris- $\mathrm{HCl}$ buffer at $\mathrm{pH}$ 7.4. Sodium dithionite $(100 \mathrm{mM})$ was used as the reducing agent. All solutions were degassed under vacuum with argon. The plug and play $(\mathrm{PnP})$ burette with chip and the $\mathrm{Ag} / \mathrm{AgCl}$ electrode were selected. Before the measurement, the burette was washed by pump ultrapure water under automatic cleaning mode program. In setting interface parameter, the titrant of sodium dithionite, the stirrer speed of $50 \%$, and the sample volume of reducing agent were set to $10 \mathrm{~mL} .10 \mathrm{~mL}$ protein sample was added to the titration cup. When the potential retained stable, sodium dithionite was titrated. Record the reduction potential and the volume of reducing agent when potential was no longer changed. 


\subsection{Construction of whole-cell catalysts system}

Whole-cell catalysts [E. coli BL21 (DE3)] with different combinations of pET28a- and pETDuet-derived vectors bearing genes encoding KshA, PRF, and Fdx were used to convert AD to 9OHAD. To construct strain BLA-PD, the PRF gene was amplified, digested, and inserted into MCS1 site of pETDuet between Eco RI andHin dIII sites, the gene encoding DmFdx2 was inserted into MCS2, between Nde I and Xho I, which created pETDuet-PRF-DmFdx2. pETDuet-PRF-DmFdx2 and pET28a harboring KshA were cotransformed into E. coli BL21(DE3) cells. The similar operation mode was used to construct strain BLAPP, in which the $P R F$ and PsFdx1 genes were inserted into pETDuet and created pETDuet-PRF-PsFdx1. pETDuet-PRF-PsFdx1 and pET28a harboring $K s h A$ were co-transformed into $E$. coli BL21(DE3) cells. The control strain of BLA-P was constructed using pET28a-KshA and pCold I-PRF, and co-transformed intoE. coli BL21(DE3) cells. The constructed strains are listed in Table S1 and plasmids are listed in Table S2.

\subsection{Bioconversion of AD to produce 9OHAD}

The constructed recombinant strains are listed in Table S1. The plasmid construction and protein expression operation are the same as described above. The cells were harvested by centrifugation at $5,000 \times \mathrm{g}$ for 10 min and resuspended in $30 \mathrm{~mL} 50 \mathrm{mM}$ PBS (pH 7.4). The production of 9OHAD was conducted in reaction mixture containing $50 \mathrm{~g} / \mathrm{L}$ wet cells, $5 \mathrm{~g} / \mathrm{L}$ AD dissolved in $2 \%$ methanol and $22.8 \mathrm{~g} / \mathrm{L}$ methyl- $\beta$-cyclodextrin, at $35{ }^{\circ} \mathrm{C}$ for $5 \mathrm{~h}$ (Zhu et al., 2020). The products were determined by HPLC.

\section{RESULTS AND DISCUSSION}

\subsection{Comparison of reductases for hydroxylation reaction toward AD}

The reductases of KshB and TDO have been screened previously with AD as the substrate (Zhu et al., 2020). A novel reported reductase of the self-sufficient P450 enzyme CYP116B46 from Tepidiphilus thermophilus (named PRF), which contains plant-type [2Fe-2S] cluster, was first mined for bioconversion of AD. We further investigated the catalytic efficiency of PRF in both $\mathrm{NAD}(\mathrm{P}) \mathrm{H}$ reduction activity and $9 \mathrm{OHAD}$ reaction system based on research on KshB and TDO (Table 1). By comparing the reduction activity toward NADH, we found that PRF showed higher activity with $k$ cat $/ K \mathrm{~m}$ of $1.70 \mathrm{~s}^{-1} \mu \mathrm{M}^{-1}$ than TDO $\left(0.43 \mathrm{~s}^{-1} \mu \mathrm{M}^{-1}\right)$ and KshB $\left(0.34 \mathrm{~s}^{-1} \mu \mathrm{M}^{-1}\right)$. Furthermore, PRF also showed the higher AD conversion rate of $68.4 \%$ than TDO (54.8\%) and KshB (42.0\%) (Figure 1 and Table S3). Therefore, PRF showed the promising potential as reductase partner of KshA during hydroxylation of steroidal substrates than KshB or TDO.

\subsection{Structural analysis of reductase TDO and PRF}

We compared the crystal structures of two reductases TDO-R (PDB ID: 3EF6) with PRF (PDB ID: 6LAA). Structural analysis revealed that both TDO and PRF contained NAD $(\mathrm{P}) \mathrm{H}$ domain, and their flavin binding motifs belonged to different types: FAD-binding and FMN-binding (Figure 2). In reductase TDO, both the FAD-binding and NADH-binding domains were $\alpha / \beta$-fold type characteristic of the glutathione reductase (GR) family. They had a central parallel $\beta$-sheet, three $\alpha$-helices on one side of the sheet and a three-stranded $\beta$-meander on the other side. FAD captured electrons from NADH and transfers to [2Fe-2S] cluster Fe atom of TDO-Fdx (Friemann et al., 2009). The structural comparison showed that the TDO contained an extra domain (gray part). In previous research, a "swing domain model" of P450BM3 FMN-binding domain operated the back-and-forth motions, which was governed by a flexible hinge domain (Chen et al., 2020). That implied that TDO-Fdx might swing to a favorable position to carry out the effective electron transfer from FAD to [2Fe-2S] cluster (Figure 2a). In PRF, the FMN binding motif was a six-stranded antiparallel $\beta$-barrel while the NADPH-binding motif adopted a Rossmann fold containing a partial $\beta$-sheet. FMN captured electrons from NADH and transferred the electrons from the methyl group of $\mathrm{C} 8$ to Fe atom of [2Fe-2S] cluster (Figure 2b) (Zhang et al., 2020). The truncated [2Fe-2S] clusters of the oxidoreductases $\left({ }^{K}\right.$ sh $\left.A,{ }^{K} \operatorname{shB},{ }^{T} D O,{ }^{P} R F\right)$ hadnolongerexhibitedcatalyticactivitytowardAD.Itindicatedthat $[2 \mathrm{Fe}-$ $2 S]$ cluster splayedtheirreplaceableroleduringthehydroxylationreactionof $A D($ Table 1$)$.Importantly, thestructuresof $[2 \mathrm{Fe}-$ $2 S]$ clustersinTDO, KshBandPRFarealsodif ferent, whichmaycausetothecompletelydif ferentcatalyticactivitiesandconve $2 S]$ clusterofTDO - FistheRieske - type(Feligatedby2Cysand2His), whilePRFandKshBaretheplant - 
type(Feligatedby4Cys).

To further investigate the importance of $[2 \mathrm{Fe}-2 \mathrm{~S}]$ clusters in reductases, we compared the catalytic activity of various reductases containing two different types of $[2 \mathrm{Fe}-2 \mathrm{~S}]$ clusters. Besides, we mutated the TDO to MTDO (plant-type). Meanwhile, KshB and PRF were mutated to MKshB and MPRF (Rieske-type), respectively. After mutation, the NADH reduction activity $k_{\text {cat }} / K_{\mathrm{m}}$ of MTDO has been increased from $0.43 \mathrm{~s}^{-1} \mu \mathrm{M}^{-1}$ to $0.54 \mathrm{~s}^{-1} \mu \mathrm{M}^{-1}$. KshB showed the same activity $\left(0.34 \mathrm{~s}^{-1} \mu \mathrm{M}^{-1}\right)$ with MKshB $\left(0.35 \mathrm{~s}^{-1} \mu \mathrm{M}^{-1}\right)$. Surprisingly, MPRF showed the decreased activity from $1.70 \mathrm{~s}^{-1} \mu \mathrm{M}^{-1}$ to $0.50 \mathrm{~s}^{-1} \mu \mathrm{M}^{-1}$ (Table 1). The data of $\mathrm{AD}$ conversion rate of three mutants also showed the same tendency with NADH reduction activity that muted MPRF also decreased the AD conversion rate (Table S3). These results implied that plant-type cluster seems to have higher electron transfer efficiency than Rieske-type cluster.

More importantly, the UV-vis absorption spectra (Figure 3a and 3b) were typical characteristics of flavoproteins. Plant-type PRF (Figure 3a) and MTDO (Figure 3b) had almost identical UV-vis spectra, with three main absorptions at 378, 447 and $475 \mathrm{~nm}$. In contrast, the wild-type TDO (Rieske-type) also showed the same main absorptions at 378 and $447 \mathrm{~nm}$, but a slight bathochromic shift of the band at $475 \mathrm{~nm}$ and a relative amplitude of the 530-550 nm shoulder (Figure $3 \mathrm{~b}$ ). This indicated that the modification of TDO (Rieske-type) to MTDO (plant-type) resulted in the slight changes in the prosthetic group environment. These small changes implied that the electronic structure of Rieske-type and plant-type [2Fe-2S] cluster was different and hence of different interactions between the two types of [2Fe-2S] clusters and the protein.

\subsection{Screening of free Fdx proteins for enhancement of steroidal conversion}

The $[2 \mathrm{Fe}-2 \mathrm{~S}]$ clusters have been testified to be important for catalytic activities. To verify whether free $\mathrm{Fdx}$ functions as electron transfer intermediate, we investigated the effects of two types of free Fdxs (Rieske-type and plant-type) according to the conversion of AD to 9OHAD using multi-enzyme catalytic system in vitro (KshA+PRF) (Figure S1and Table S4). Six Fdxs genes were screened as candidates namely PsFdx1, SyFdx, PrFdx (Rieske-type Fdxs) and TeFdx, DmFdx1, DmFdx2 (Plant-type Fdxs).

After adding various free Fdxs in the 9OHAD catalytic reaction system containing reductase PRF and oxygenase KshA, the AD conversion rate were determined and the Fdxs candidate enzymes were evaluated. The most outstanding in six Fdxs are Rieske-type PsFdx1 and plant-type DmFdx2 (Figure S1). Therefore, we selected these two Fdxs for further bioconversion of AD to 9OHAD in oxidoreductase system using reductases KshB, TDO and PRF. In particularly, when adding Rieske-type Fdx PsFdx1 into the catalytic reaction system, the $\mathrm{AD}$ conversion rates of $\mathrm{KshA}+\mathrm{PRF}+\mathrm{PsFdx} 1$ and $\mathrm{KshA}+\mathrm{PRF}+\mathrm{DmFdx} 2$ were $80.6 \%$ and 85.2\% (Figure S2a), which was 1.18 and 1.25 folds higher than only using KshA+PRF (68.4\%) respectively. The reaction systems of TDO and KshB also showed the similar increase of AD conversion rates when adding two types of Fdx (Figure S2b and S2c). When free Fdx was added to the catalytic reaction system, the electron transfer was effectively promoted due to the mediation of free $\mathrm{Fdx}$ among reductase and oxygenase. Furthermore, the plant-type DmFdx2 was more efficient than Rieske-type PsFdx1, when adding these two different kinds of Fdxs to catalytic reaction system.

\subsection{Structuralanalysis of two types ofFdxs}

All the six free Fdxs are [2Fe-2S] clusters, which can be divided into plant-type and Rieske-type according to the difference of coordinating amino acids in the [2Fe-2S] cluster (Figure S3). Sequence alignment showed that all the Rieske-type Fdxs have conserved Cys-X1-His-segment-Cys-X2-His motif (Figure S4a). N-terminal portion of the Rieske-type Fdx consists of a Rieske [2Fe-2S] cluster domain, which consists of $\beta$ strands and loops (Ferraro et al., 2007; Murzin et al., 1995). Four residues in the $\alpha$ subunit, 2 His and 2Cys, coordinate the Rieske non-heme iron in $[2 \mathrm{Fe}-2 \mathrm{~S}]$ cluster. $2 \mathrm{His}$ coordinate one iron, while $2 \mathrm{Cys}$ coordinate the other (Figure S3a, b, c). At the same time, all the plant-type Fdxs have conservative Cys-X4/X5-Cys-X2-Cyssegment-Cys motif (Figure S4b). The C-terminal portion consists of a plant-type [2Fe-2S] cluster domain, which consists of loops, $\beta$ strands and $\alpha$ spiral. Four residues of Cys in the $\alpha$ subunit coordinate two irons of non-heme iron cluster [2Fe-2S] (Figure S3d, e, f). 
The UV-vis spectroscopic absorption of all oxidized Fdxs showed characteristic absorption spectra in range of 300-800 nm. DmFdx2 (Figure 3c) and PsFdx1 (Figure 3d) showed distinguished different UV-Vis spectra. DmFdx2 showed typical spectrum of plant-type Fdxs with three main absorptions at 330, 415 and $465 \mathrm{~nm}$. After reduction with sodium dithionite, the absorption decreased by $52 \%$ at $420 \mathrm{~nm}$ and $49 \%$ at $465 \mathrm{~nm}$. The UV-vis absorption spectrum of Rieske-type PsFdx1 (Figure 3b) showed bands at 330 and $450 \mathrm{~nm}$, but a relative amplitude of the 550-580 $\mathrm{nm}$ shoulder. When reduced with sodium dithionite, the absorbance of PsFdx1 decreased in the entire UV-visible range. These typical features of two types [2Fe-2S] Fdxs indicated that the electronic structure of Rieske-type and plant-type $[2 \mathrm{Fe}-2 \mathrm{~S}]$ cluster was different.

\subsection{Structural analysis of proposed electron transfer pathway}

To analyze the electron transfer pathway between free $\mathrm{Fdx}$, reductase and oxygenase, we targeted the reaction system of the KshA (oxygenase), PRF (reductase) and DmFdx2 (Fdx) to investigate the electron transfer pathway using protein-protein docking (Figure S5). Interestingly, the docked structure complex revealed that there were two potential electron transfer pathways existing in the reaction system. Adding free Fdx creates a new conduit for electrons to travel from reductase to oxygenase. In the KshA+PRF catalytic reaction system, the electron transfer pathway is PRF (NADPH-FMN-[2Fe-2S]) - KshA ([2Fe-2S] -Fe). After free Fdx was added to the catalytic reaction system, an auxiliary electron transfer pathway of PRF(NADPH-FMN$[2 \mathrm{Fe}-2 \mathrm{~S}])-\mathrm{DmFdx} 2([2 \mathrm{Fe}-2 \mathrm{~S}])$ - KshA ([2Fe-2S] -Fe) might be formed between PRF and DmFdx2, in which the electrons could also be transferred to the [2Fe-2S] of free $\mathrm{Fdx}$, and then were captured by the [2Fe-2S] of the PRF. The physical distance between two [2Fe-2S] clusters of PRF and KshA $(20.9 \AA)$ was almost the same with the distance between $[2 \mathrm{Fe}-2 \mathrm{~S}]$ clusters of PRF and DmFdx2 $(20.4 \AA)$. It seems that electrons have the same probability to be transferred by both pathways (Figure S5). Furthermore, the distance between [2Fe-2S] clusters of DmFdx2 and KshA was only $9.3 \AA$, implied that electrons could be transferred more effectively to KshA. The structural information implied that two electron transfer pathways might be more efficient for improving the $\mathrm{AD}$ conversion rate.

\subsection{Analysis of protein-protein interactions identified by ITC}

To further identify the proposed electron transfer pathway of free Fdx as an electron carrier, the interactions of DmFdx2 with KshA and PRF were measured via thermodynamics ITC experiments. The results of the calorimetric titrations of DmFdx2 to PRF (Figure 4a) and DmFdx2 to KshA (Figure 4b) were shown in Figure 4 . The thermodynamic parameters were collected in Table 2. In both cases, an exothermic heat pulse $(\Delta H<0)$ was observed after each injection of DmFdx2 into the aptamer solution KshA and PRF. This indicated that the binding process was an exothermic reaction. However, the interaction of PRF with DmFdx2 was associated with a larger favorable enthalpy $\left({ }^{H}=-8 \mathrm{KJmol}^{-1}\right) \operatorname{comparedwithK} \operatorname{sh} A\left({ }^{H}=\right.$

$\left.-6 \mathrm{KJmol}^{-1}\right)$.ThevaluesofthebindingconstantsandtheGibbsenergychangesindicatedthattheassociationswerestrongly favor $\left.2.10 \times 10^{7} M^{-1}\right)$ isabout $1.5-$ foldgreaterthanthatforthePRF $-\operatorname{DmFdx2interaction}\left(K_{D}=\right.$ $\left.1.50 \times 10^{7} M^{-1}\right)$.Thevaluesofentropychange $\left({ }^{S}<0\right)$ showedthat, inbothcases, thebindingprocesseswereenthalpicallydriven.The

\subsection{Analysis of redox potential and electron bifurcation}

To clarify the auxiliary electron transfer pathway between two [2Fe-2S] clusters of reductase PRF and ferredoxin DmFdx2, we performed the measurement of the redox potential (Table 2). The redox potential measurement indicated that the single-pathway potential and the dual-pathway potential are different. Particularly, single-pathway showed the redox potential of PRF $(-365 \mathrm{mV})$ and KshA $(-346 \mathrm{mV})$. However, DmFdx2 had lower reduction potential $(-390 \mathrm{mV})$ and higher reduction ability in dual-pathway. It might be reasonable that the lower the free energy of the final state, the higher would be the electron transfer efficiency. After the PRF captures the two electrons from the FMN, one electron with a lower energy level is directly transferred to the oxygenase KshA, and the other electron with a higher energy level is transferred to reduce DmFdx2 (Figure 5). Compared with the potential difference of PRF (-365mV) and KshA (-346mV), the larger potential difference between $\operatorname{PRF}(-365 \mathrm{mV})$ and $\mathrm{DmFdx} 2(-390 \mathrm{mV})$ has more enery and tendency to transfer electrons. Furthermore, the distance between the two [2Fe-2S] clusters of DmFdx2 and oxygenase KshA was only 9.3A, which seems to transfer electrons more effectively (Figure S5). The analysis of redox 
potential and electron bifurcation was consistent with the bioconversion of $\mathrm{AD}$ that adding $\mathrm{Fdx}$ into the catalytic system might improve conversion rate.

The redox potentials also indicated that PRF $(-365 \mathrm{mV})$ has lower redox potential than $\mathrm{KshB}(-352 \mathrm{mV})$. It implied that reductase PRF had stronger redox capacity and higher biotransformation capacity than KshB. In addition, by using free $\mathrm{Fdx}$ to titrate the mixed system of reductase and oxygenase, we found that the final titration states showed lower redox potential of PRF+KshA $(-94 \mathrm{mV})$ than $\mathrm{KshB}+\mathrm{KshA}(-80 \mathrm{mV})$. All these redox potential data explain excellent ability in steroid transformation of $\mathrm{PRF}+\mathrm{Fdx}+\mathrm{KshA}$ reaction system.

\subsection{Bioconversion of AD to produce 9OHAD}

Whole-cell catalysis systems were constructed in the E. coliBL21(DE3) for stable and sustainable production of 9OHAD. We constructed three industrial strains of BLA-PD, BLA-PP and BLA-P. Whole-cell catalytic system without free Fdxs of BLA-P (KshA+PRF-BL21) only showed the yield of 9OHAD with $76.7 \%$ in $5 \mathrm{~h}$. However, the whole-cell catalytic system with free Fdxs of BLA-PD (KshA+PRF+DmFdx2-BL21) showed the highest yield of 9OHAD with $(95.4 \%)$ in $5 \mathrm{~h}$, followed by BLA-PP (KshA+PRF+PsFdx1-BL21) (90.2\%). This implied that add free Fdxs, especially plant-type DmFdx2, attributed to increase the electron transfer efficiency in the catalytic system of KshA+PRF. It also implied the importance of the predominant second electron transfer pathway mentioned above.

In comparison, the engineered Mycobacterium neoaurum ATCC 25795 reached a $54.5 \%$ yield of $9 \mathrm{OHAD}$ from $20 \mathrm{~g} / \mathrm{L}$ phytosterol in $72 \mathrm{~h}$ as substrate by modifying key genes related to metabolism to increase cell permeability (Xiong et al., 2020). The Rhodococcus erythropolisRG1-UV29 strain reached a $93 \%$ yield of $9 \mathrm{OHAD}$ from $20 \mathrm{~g} / \mathrm{L} \mathrm{AD}$ in $24 \mathrm{~h}$ (Geize et al., 2001). However, the recombinant E. coli only reached a $63 \%$ yield of 9OHAD from $1 \mathrm{~g} / \mathrm{L} \mathrm{AD}$ in $48 \mathrm{~h}$ (Petrusma et al., 2009). Therefore, the constructed whole-cell catalytic system of BLA-PD with free Fdxs exhibited an excellent catalytic ability, and has the potential to be used industrially for efficient 9OHAD production.

In our previous research, Zhu et al. constructed E. coliBL21(DE3) strain of BLKA-RTM-F, which contained the NADH recycling system and a modified TDO mutant with two Rieske-type [2Fe-2S] clusters at the N/C-terminus. It exhibited excellent 9OHAD productivity with $5.24 \mathrm{~g} / \mathrm{L}$ from $5 \mathrm{~g} / \mathrm{L} \mathrm{AD}$ and a yield of $99.3 \%$ without by-products. ${ }^{18}$ However, BLA-PD (KshA+PRF+DmFdx2-BL21) constructed in the present work showed the highest $\mathrm{AD}$ conversion rate of $95.4 \%$, slightly lower than BLKA-RTM-F. It may be due to the construction of NADH recycling system in BLKA-RTM-F, because NADH could be completely consumed in about 16 min. It was thought to be a limiting factor. The future work would focus on the NADH recycling system combined with the current whole-cell catalysts to improve the AD conversion rate and sustainability.

\section{CONCLUSIONS}

We screened the reductase PRF with high NADPH reduction activity and AD conversion rate. The screened plant-type DmFdx2 could strengthen the biotransformation of AD due to the high electron transfer efficiency. Based on the electron bifurcation theory, we inferred that free Fdx created a new conduit for electrons to travel from reductase to oxygenase. The protein-protein structure docking and redox potential measurement had proven this speculation. The thermodynamic data of ITC also indicated the rationality of the new and efficient electron transfer method using exogenous $\mathrm{Fdx}$ as an effective electron carrier. Whole-cell catalytic system with free Fdxs of BLA-PD (KshA+PRF+DmFdx2-BL21) showed a 95.4\% yield of 9OHAD indicating the importance of effective electron transfer carrier and a predominant reductase with high activity.

\section{ABBREVIATIONS}

9OHAD, 9 $\alpha$-hydroxy4-androstene-3,17-dione; AD, 4-androstene-3,17-dione; KshA, Rieske-type oxygenase of 3-ketosteroid-9-hydroxylase; KshB, pant-type reductase of 3-ketosteroid-9-hydroxylase; TDO, toluene 2,3dioxygenase reductase; PRF, self-sufficient P450 CYP116B46 (V465-L779) from Tepidiphilus thermophilus ; Fdx, ferredoxin; TeFdx, Thermosynechococcus elongatus BP-1 Fdx; DmFdx1,Drosophila melanogaster Fdx1; 
DmFdx2, Drosophila melanogaster Fdx2; PrFdx, Pseudomonas resinovorans Fdx; PsFdx1,Pseudomonas sp. Fdx; SyFdx, Sphingobium yanoikuyae Fdx.

\section{ACKNOWLEDGEMENTS}

This work was supported by the National Key Research and Development Program of China, Synthetic Biology Research (Grant No. 2019YFA0905300), National Natural Science Foundation of China (31771911 and 21878233), and Tianjin Synthetic Biotechnology Innovation Capacity Improvement Project (TSBICIPKJGG-001-09).

\section{CONFLICT OF INTERESTS}

The authors declare that there are no conflict of interests.

\section{DATA AVAILABILITY STATEMENT}

The data that support the findings of this study are available from the corresponding author upon reasonable request.

\section{AUTHORS' CONTRIBUTIONS}

Zhan Song: Performed the experiments, Data analysis, Writing - original draft. Cancan Wei, Chao Li and Xin Gao: Performed the experiments, Data analysis. Shuhong Mao, Fuping Lu and Hui-Min Qin: Designed the research, Writing - review \& editing, Supervision. All authors read and approved the final manuscript.

\section{Corresponding Author}

S. Mao: shuhongmao@tust.edu.cn;

F. Lu: lfp@tust.edu.cn;

H.-M. Qin: huiminqin@tust.edu.cn.

\section{REFERENCES}

Aono, S., Kurita, H., Uno, S., \& Okura, I. (1992). Purification and characterization of a 7Fe ferredoxin from a thermophilic hydrogen-oxidizing bacterium, bacillus schlegelii. The Journal of Biochemistry, 112 , 792-795. https://doi.org/10.1093/oxfordjournals.jbchem.a123977

Arnon, D. I. (1965). Ferredoxin and photosynthesis. Science, 149 , 1460-1470. https://doi.org/10.1126/science.149.3691.1460

Arnon, D. I. (1988). The discovery of ferredoxin: the photosynthetic path. Reflections Biochemistry, 13 , 30-33. https://doi.org/10.1016/0968-0004(88)90016-3

Bell, S. G., McMillan, J. H., Yorke, J. A., Kavanagh, E., Johnson, E. O., \& Wong, L. L. (2012). Tailoring an alien ferredoxin to support native-like P450 monooxygenase activity. Chemical Communications, 48 (95), 11692-11694. https://doi.org/10.1039/c2cc35968e

Bohme, H. (1977). On the role of ferredoxin and ferredoxin-NADP+ reductase in cyclic electron transport of spinach chloroplasts.European Journal of Biochemistry, 72 , 283-289. https://doi.org/10.1111/j.14321033.1977.tb11251.x

Chen, C. C., Min, J., Zhang, L., Yang, Y., Yu, X., \& Guo, R. T. (2020). Advanced understanding of the electron transfer pathway of cytochrome P450s. Chembiochem . https://doi.org/10.1002/cbic.202000705

Demmer, J. K., Huang, H., Wang, S., Demmer, U., Thauer, R. K., \& Ermler, U. (2015). Insights into flavin-based electron bifurcation via the NADH-dependent reduced ferredoxin:NADP oxidoreductase structure.Journal of Biological Chemistry, 290 (36), 21985-21995. https://doi.org/10.1074/jbc.M115.656520 
Duan, H. D., Mohamed-Raseek, N., \& Miller, A. F. (2020). Spectroscopic evidence for direct flavin-flavin contact in a bifurcating electron transfer flavoprotein. Journal of Biological Chemistry, 295 (36), 1261812634. https://doi.org/10.1074/jbc.RA120.013174

Ferraro, D. J., Brown, E. N., Yu, C.-L., Parales, R. E., Gibson, D. T., \& Ramaswamy, S. (2007). Structural investigations of the ferredoxin and terminal oxygenase components of the biphenyl 2,3-dioxygenase from Sphingobium yanoikuyae B1. BMC Structural Biology, 7 , 10. https://doi.org/10.1186/1472-6807-7-10

Friemann, R., Lee, K., Brown, E. N., Gibson, D. T., Eklunda, H., \& Ramaswamyd. (2009). Structures of the multicomponent Rieske non-heme iron toluene 2,3-dioxygenase enzyme system. Acta Crystallographica. Section D, Biological Crystallography, 65 (Pt 1), 24-33. https://doi.org/10.1107/S0907444908036524

Geize, R. v. d., Hessels, G. I., Gerwen, R. v., Meijden, P. v. d., \& Dijkhuizen, L. (2001). Unmarked gene deletion mutagenesis of kstD, encoding 3ketosteroid $\quad 1 \quad-\quad$ dehydrogenase, inRhodococcuserythropolisSQ1usingsacBascounter selectablemarker.FEMS Microbiology Letters, 205,197 - 202.https : $\quad / /$ doi.org/10.1111/j.1574 6968.2001.tb10947.x

Goss, T., \& Hanke, G. (2014). The end of the line: can ferredoxin and ferredoxin NADP(H) oxidoreductase determine the fate of photosynthetic electrons? Current Protein $\mathcal{E}$ Peptide Science, 15 , 385-393. https://doi.org/10.2174/1389203715666140327113733

Huang, H., Hu, L., Yu, W., Li, H., Tao, F., Xie, H., \& Wang, S. (2016). Heterologous overproduction of $2[4 \mathrm{Fe} 4 \mathrm{~S}]-$ and $[2 \mathrm{Fe} 2 \mathrm{~S}]$-type clostridial ferredoxins and [2Fe2S]-type agrobacterial ferredoxin.Protein Expression and Purification, 121 , 1-8. https://doi.org/10.1016/j.pep.2015.12.019

Hurley, J. K., Weber-Main, A. M., Stankovich, M. T., Benning, M. M., Thoden, J. B., Vanhooke, J. L., . . . Tollin, G. (1997). Structure-function relationships in anabaena ferredoxin: correlations between X-ray crystal structures, reduction potentials, and rate constants of electron transfer to ferredoxin: NADP + reductase for site-specific ferredoxin mutants. Biochemistry, 36 , 11100-11117. https://doi.org/10.1021/bi9709001

Imai, T., Taguchi, K., Ogawara, Y., Ohmor, D., Yamakura, F., Ekezawa, H., \& Urushiyama, A. (2001). Characterization and cloning of an extremely thermostable, Pyrococcus furiosus-type $4 \mathrm{Fe}$ ferredoxin from Thermococcus profundus. The Journal of Biochemistry, 130 , 649-655. https://doi.org/10.1093/oxfordjournals.jbchem.a003030

Kameya, M., Arai, H., \& Ishii, M. (2020). Importance of electron flow in microbiological metabolism .

Kimata-Ariga, Y., Kurisu, G., Kusunoki, M., Aoki, S., Sato, D., Kobayashi, T., Hase, T. (2006). Cloning and characterization of ferredoxin and ferredoxin-NADP+ reductase from human malaria parasite. The Journal of Biochemistry, 141 (3), 421-428. https://doi.org/10.1093/jb/mvm046

Kimata-Ariga, Y., Saitoh, T., Ikegami, T., Horii, T., \& Hase, T. (2007). Molecular interaction of ferredoxin and ferredoxin-NADP + reductase from human malaria parasite. The Journal of Biochemistry, 142 (6), 715-720. https://doi.org/10.1093/jb/mvm184

Kremp, F., Roth, J., \& Muller, V. (2020). The Sporomusa type Nfn is a novel type of electron-bifurcating transhydrogenase that links the redox pools in acetogenic bacteria. Scientific Reports, 10 (1), 14872. https://doi.org/10.1038/s41598-020-71038-2

Lee, Y.-H., Ikegami, T., Standley, D. M., Sakurai, K., Hase, T., \& Goto, Y. (2011). Binding energetics of ferredoxin-NADP + reductase with ferredoxin and its relation to function. Chembiochem, 12 (13), 2062-2070. https://doi.org/10.1002/cbic.201100189

Liang, J., Huang, H., \& Wang, S. (2019). Distribution, evolution, catalytic mechanism, and physiological functions of the flavin-based electron-bifurcating NADH-dependent reduced ferredoxin: NADP+ oxidoreductase. Frontiers in Microbiology, 10 , 373. https://doi.org/10.3389/fmicb.2019.00373 
Lovgreen, M. N., Martic, M., Windahl, M. S., Christensen, H. E., \& Harris, P. (2011). Crystal structures of the all-cysteinyl-coordinated D14C variant of Pyrococcus furiosus ferredoxin: $[4 \mathrm{Fe}-4 \mathrm{~S}]<\longrightarrow[3 \mathrm{Fe}-4 \mathrm{~S}]$ cluster conversion. Journal of Biological Inorganic Chemistry, 16 (5), 763-775. https://doi.org/10.1007/s00775-0110778-7

Lubner, C. E., Jennings, D. P., Mulder, D. W., Schut, G. J., Zadvornyy, O. A., Hoben, J. P., Peters, J. W. (2017). Mechanistic insights into energy conservation by flavin-based electron bifurcation. Nature Chemical Biology, 13 (6), 655-659. https://doi.org/10.1038/nchembio.2348

Martı'nez-Espinosa, R. M., Marhuenda-Egea, F. C., Donaire, A., \& Bonetea, M. a. J. (2003). NMR studies of a ferredoxin from Haloferax mediterranei and its physiological role in nitrate assimilatory pathway.Biochimica et Biophysica Acta, 1623 (1), 47-51. https://doi.org/10.1016/S0304-4165(03)00157-0

Miller, A. F., Duan, H. D., Varner, T. A., \& Mohamed Raseek, N. (2019). Reduction midpoint potentials of bifurcating electron transfer flavoproteins. Methods in Enzymology, 620 , 365-398. https://doi.org/10.1016/bs.mie.2019.03.017

Mortenson, L. E., Valentine, R. C., \& Carnahan, J. E. (1962). An electron transport factor from Closiriium pasteurianum.Biochemical and Biophysical Research Communications, 7 (6), 448-452. https://doi.org/10.1016/0006-291X(62)90333-9

Muller, V., Chowdhury, N. P., \& Basen, M. (2018). Electron Bifurcation: A Long-Hidden Energy-Coupling Mechanism. Annual Review of Microbiology, 72 , 331-353. https://doi.org/10.1146/annurev-micro-090816093440

Murzin, A. G., Brenner, S. E., Hubbard, T., \& Chothia, C. (1995). SCOP: A Structural classification of proteins database for the investigation of sequences and structures. Journal of Molecular Biology, 247, 536-540. https://doi.org/10.1016/S0022-2836(05)80134-2

Petrusma, M., Dijkhuizen, L., \& van der Geize, R. (2009). Rhodococcus rhodochrous DSM 43269 3-ketosteroid 9alpha-hydroxylase, a two-component iron-sulfur-containing monooxygenase with subtle steroid substrate specificity. Applied and Environmental Microbiology, 75 (16), 5300-5307. https://doi.org/10.1128/AEM.00066-09

Quaranta, A., Lagoutte, B., Frey, J., \& Sétif, P. (2016). Photoreduction of the ferredoxin/ferredoxin-NADP+reductase complex by a linked ruthenium polypyridyl chromophore. Journal of Photochemistry and Photobiology B: Biology, 160 , 347-354. https://doi.org/10.1016/j.jphotobiol.2016.04.030

Ricagno, S., de Rosa, M., Aliverti, A., Zanetti, G., \& Bolognesi, M. (2007). The crystal structure of FdxA, a 7Fe ferredoxin from Mycobacterium smegmatis. Biochemical and Biophysical Research Communications, 360 (1), 97-102. https://doi.org/10.1016/j.bbrc.2007.06.013

Schut, G. J., Mohamed-Raseek, N., Tokmina-Lukaszewska, M., Mulder, D. W., Nguyen, D. M. N., Lipscomb, G. L., . . . Adams, M. W. W. (2019). The catalytic mechanism of electron-bifurcating electron transfer flavoproteins (ETFs) involves an intermediary complex with NAD+.Journal of Biological Chemistry, 294 (9), 3271-3283. https://doi.org/10.1074/jbc.RA118.005653

Senda, M., Kishigami, S., Kimura, S., Fukuda, M., Ishida, T., \& Senda, T. (2007). Molecular mechanism of the redox-dependent interaction between NADH-dependent ferredoxin reductase and Rieske-type [2Fe-2S] ferredoxin. Journal of Molecular Biology, 373 (2), 382-400. https://doi.org/10.1016/j.jmb.2007.08.002

Sucharitakul, J., Buttranon, S., Wongnate, T., Chowdhury, N. P., Prongjit, M., Buckel, W., \& Chaiyen, P. (2020). Modulations of the reduction potentials of flavin-based electron bifurcation complexes and semiquinone stabilities are key to control directional electron flow.FEBS Journal, 288 (3), 1008-1026. https://doi.org/10.1111/febs.15343

Tokmina-Lukaszewska, M., Patterson, A., Berry, L., Scott, L., Balasubramanian, N., \& Bothner, B. (2018). The role of mass spectrometry in structural studies of flavin-based electron bifurcating enzymes. Frontiers 
in Microbiology, 9 , 1397. https://doi.org/10.3389/fmicb.2018.01397

Ueno, Y., Matsumoto, T., Yamano, A., Imai, T., \& Morimoto, Y. (2013). Increasing the electron-transfer ability of Cyanidioschyzon merolae ferredoxin by a one-point mutation-a high resolution and Fe-SAD phasing crystal structure analysis of the Asp58Asn mutant.Biochemical and Biophysical Research Communications, 436 (4), 736-739. https://doi.org/10.1016/j.bbrc.2013.06.029

Xiong, L. B., Liu, H. H., Zhao, M., Liu, Y. J., Song, L., Xie, Z. Y., . . . Wei, D. Z. (2020). Enhancing the bioconversion of phytosterols to steroidal intermediates by the deficiency of kasB in the cell wall synthesis of Mycobacterium neoaurum. Microbial Cell Factories, 19 (1), 80. https://doi.org/10.1186/s12934-020-01335y

Yuly, J. L., Lubner, C. E., Zhang, P., Beratan, D. N., \& Peters, J. W. (2019). Electron bifurcation: progress and grand challenges. Chemical Communications, 55 (79), 11823-11832. https://doi.org/10.1039/c9cc05611d

Zhang, L., Xie, Z., Liu, Z., Zhou, S., Ma, L., Liu, W., . . . Chen, C. C. (2020). Structural insight into the electron transfer pathway of a self-sufficient P450 monooxygenase. Nature Communications, 11 (1), 2676. https://doi.org/10.1038/s41467-020-16500-5

Zhu, Z., Gao, X., Song, Z., Li, C., Lu, F., Tanokura, M., \& Qin, H.-M. (2020). Development of engineered ferredoxin reductase ssystems for the efficient hydroxylation of steroidal substrates. ACS Sustainable Chemistry \& Engineering, 8 (44), 16720-16730. https://doi.org/10.1021/acssuschemeng.0c07042

Table 1 Kinetic parameters of reductases and ferredoxin toward NADH

\begin{tabular}{llllll}
\hline Reductase & $K_{\mathrm{m}}(\mu \mathrm{M})$ & $k_{\text {cat }}\left(\mathrm{s}^{-1}\right)$ & $k_{\text {cat }}\left(\mathrm{s}^{-1}\right)$ & $k_{\text {cat }} / K_{\mathrm{m}}\left(\mathrm{s}^{-1} \mu \mathrm{M}^{-1}\right)$ & $k_{\text {cat }} / K_{\mathrm{m}}\left(\mathrm{s}^{-1} \mu \mathrm{M}^{-1}\right)$ \\
\hline TDO* & $65.37 \pm 2.71$ & $65.37 \pm 2.71$ & $28.21 \pm 0.91$ & $28.21 \pm 0.91$ & 0.43 \\
${ }^{T} D O$ & $/$ & $/$ & $/$ & $/$ & $/$ \\
MTDO & $63.53 \pm 2.95$ & $63.53 \pm 2.95$ & $34.27 \pm 0.69$ & $34.27 \pm 0.69$ & 0.54 \\
KshB* & $70.85 \pm 4.08$ & $70.85 \pm 4.08$ & $23.88 \pm 1.06$ & $23.88 \pm 1.06$ & 0.34 \\
${ }^{K}$ shB & $/$ & $/$ & $/$ & $/$ & $/$ \\
MKshB & $69.94 \pm 2.12$ & $69.94 \pm 2.12$ & $24.13 \pm 0.89$ & $24.13 \pm 0.89$ & 0.35 \\
PRF & $39.11 \pm 1.49$ & $39.11 \pm 1.49$ & $66.43 \pm 0.34$ & $66.43 \pm 0.34$ & 1.70 \\
${ }_{P} R F$ & $/$ & $/$ & $/$ & $/$ & $/$ \\
MPRF & $63.12 \pm 2.37$ & $63.12 \pm 2.37$ & $31.27 \pm 1.17$ & $31.27 \pm 1.17$ & 0.50 \\
\hline
\end{tabular}

Note: Data are shown as the mean \pm SD from three independent experiments.

* data from Reference Zhu et al.

Table 2 Thermodynamic parameters for the interaction with DmFdx2

\begin{tabular}{|c|c|c|c|c|c|}
\hline & $n$ & $K_{D}\left(\mathrm{M}^{-1}\right)$ & ${ }^{H}\left(K J m o l^{-1}\right)$ & ${ }^{S}\left(K_{J m o l}{ }^{-1} K^{-1}\right)$ & $G_{298 \mathrm{k}}\left(\mathrm{KJmol}^{-1}\right)$ \\
\hline PRF & $0.75 \pm 0.01$ & $\begin{array}{l}(1.50 \pm 0.10) \\
\times 10^{7}\end{array}$ & $-8 \pm 0.82$ & $0.11 \pm 0.02$ & $-40.94 \pm 1$ \\
\hline $\mathrm{K} \operatorname{sh} \mathrm{A}$ & $0.40 \pm 0.02$ & $\begin{array}{l}(2.10 \pm 0.10) \\
\times 10^{7}\end{array}$ & $-6 \pm 0.13$ & $0.12 \pm 0.03$ & $-41.81 \pm 1$ \\
\hline
\end{tabular}

Table 3 Redox potential of reductase and ferredoxin

\begin{tabular}{llll}
\hline Enzymes(V1) & Titrant(V2) & Volume ratio (V1:V2) & Electric potential $(\mathrm{mV})$ \\
\hline KshA & $\mathrm{Na}_{2} \mathrm{~S}_{2} \mathrm{O}_{4}$ & $2: 1$ & $-346 \pm 1$
\end{tabular}




\begin{tabular}{llll}
\hline Enzymes(V1) & Titrant(V2) & Volume ratio (V1:V2) & Electric potential $(\mathrm{mV})$ \\
\hline KshB & $\mathrm{Na}_{2} \mathrm{~S}_{2} \mathrm{O}_{4}$ & $2: 1$ & $-352 \pm 1$ \\
PRF & $\mathrm{Na}_{2} \mathrm{~S}_{2} \mathrm{O}_{4}$ & $2: 1$ & $-365 \pm 2$ \\
DmFdx2 & $\mathrm{Na}_{2} \mathrm{~S}_{2} \mathrm{O}_{4}$ & $2: 1$ & $-390 \pm 1$ \\
KshB+KshA & $\mathrm{DmFdx}_{2}$ & $1: 3$ & $-80 \pm 3$ \\
PRF+KshA & DmFdx2 & $1: 3$ & $-94 \pm 1$ \\
\hline
\end{tabular}

\section{FIGURE LEGENDS}

Figure 1. Conversion rate of AD to 9OHAD. AD conversion rate of various reductases of KshB, PRF, TDO or mutants MKshB, MPRF, MTDO in KshA+reductase catalytic reaction system.

Figure 2. Structural comparison of reductase domain of TDO and PRF. The cartoon and surface representation of the reductase domain of TDO (a) and PRF (b). NADH binding domain is shown in sallow and FAD/FMN binding domain is shown in pink, FAD and FMN are represented by sticks.

Figure 3. UV-vis absorption spectrum of Fdxs and reductase. The UV-vis absorption spectra of the oxidation state and sodium dithionite-reduced are black and red respectively, and shown as (a) reductase PRF, (c)DmFdx2 (plant-type) and (d)PsFdx1 (Reiske-type). (b)Comparison of the UV-vis absorption spectra of wild-type TDO (blue) and mutant MTDO (green).

Figure 4. The affinity measurements between DmFdx2 and PRF/KshA by ITC. The affinity of reductase PRF (a) and oxygenase KshA (b) (0.2 mM DmFdx2 vs $0.02 \mathrm{mM} \mathrm{PRF/KshA)} \mathrm{to} \mathrm{DmFdx2} \mathrm{was}$ determined by ITC 200 in $20 \mathrm{mM}$ HEPES buffer (pH 7.4) at $25^{\circ} \mathrm{C}$. The raw data of the calorimetric titration and the curve fit of the observed heat are displayed in the upper and lower panels, respectively.

Figure 5. Analysis of the proposed electron transfer pathway between oxidoreductase and free Fdx. The overall structures of reductase PRF, free ferredoxin DmFdx2 and oxygenase KshA are shown as surface representation. NADH and FMN are shown as sticks, iron atoms and [2Fe-2S] cluster groups are shown as spheres.

Figure 1. 


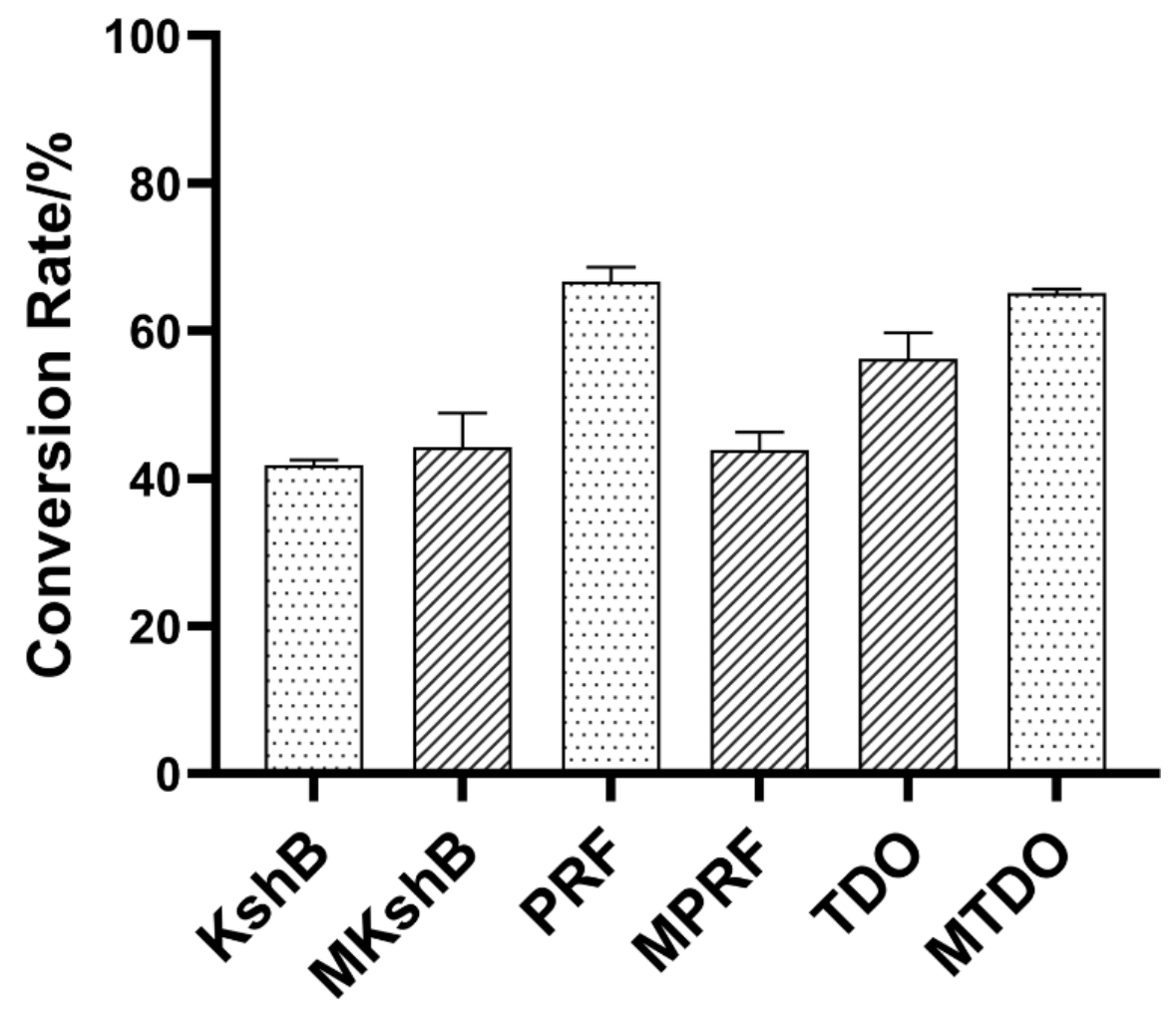

Figure 2.

a

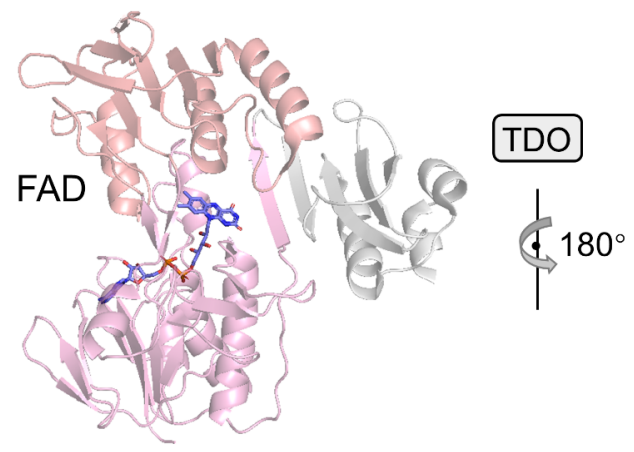

b

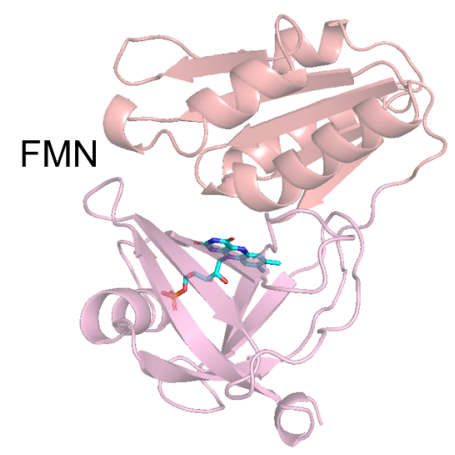

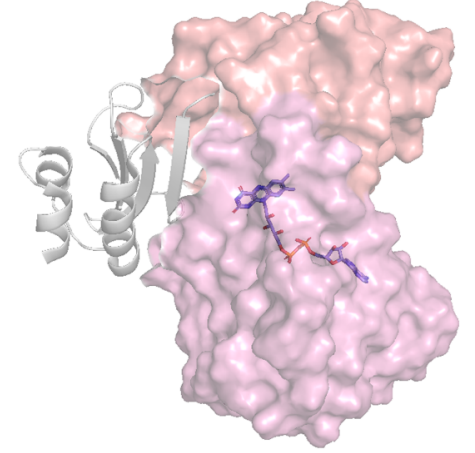

PRF
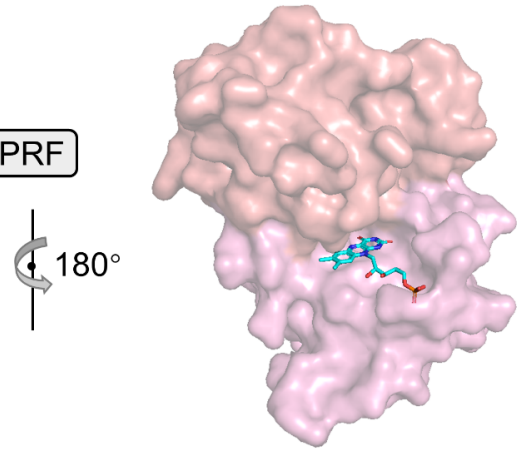

Figure 3. 

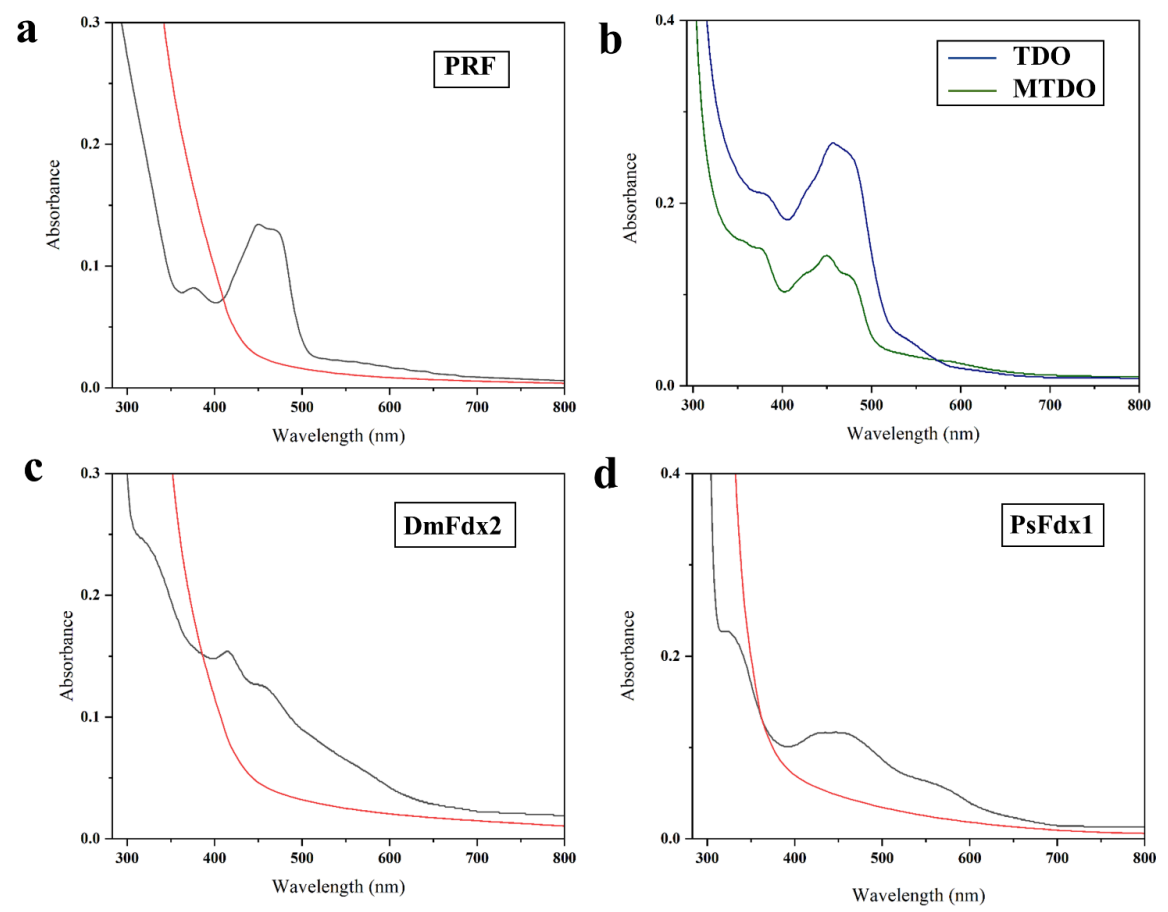

Figure 4 .
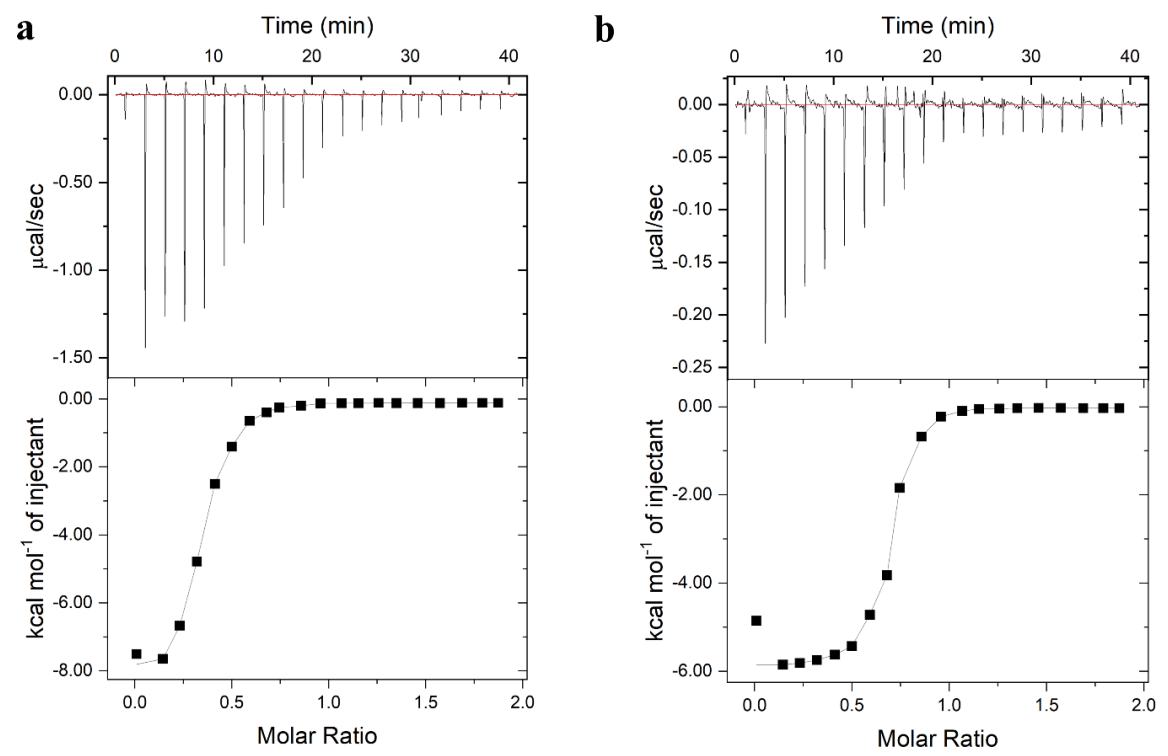

Figure 5. 


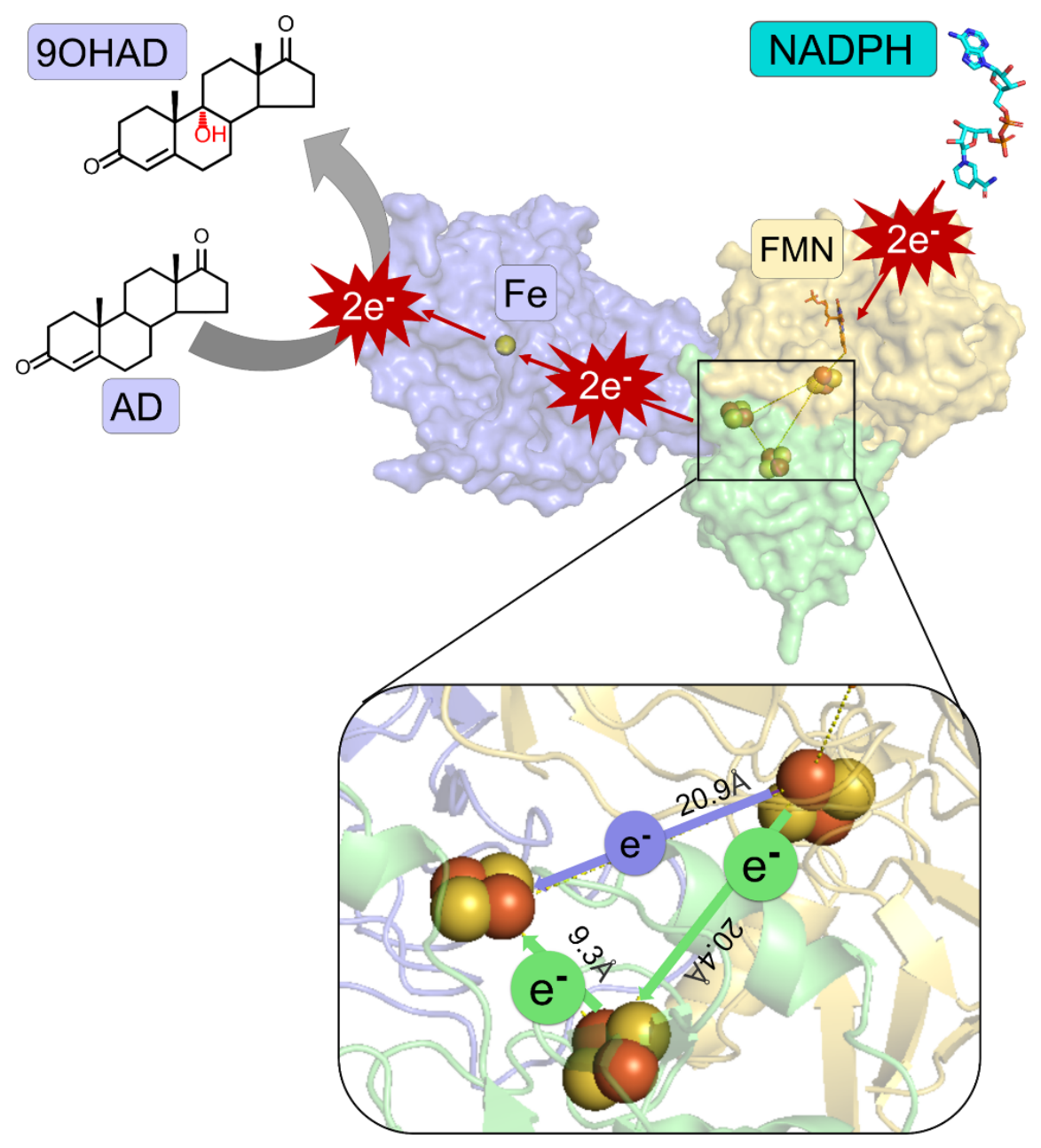

